\title{
Targeting Myocardial Reperfusion Injury — The Search Continues
}

\author{
Derek J. Hausenloy, M.B., Ch.B., Ph.D., and Derek M. Yellon, D.Sc.
}

Timely reperfusion by means of primary percutaneous coronary intervention (PCI) is the most effective therapy for limiting myocardial infarct size in patients with ST-segment elevation myocardial infarction (STEMI). Although mortality in this patient population is falling, the number of patients in whom heart failure develops is increasing. As such, new treatments are needed to reduce myocardial infarct size and to preserve left ventricular function, so as to prevent the onset of heart failure.

One neglected therapeutic target is myocardial reperfusion injury, the term given to the cardiomyocyte death that paradoxically results from reperfusion of ischemic myocardium. ${ }^{1}$ This phenomenon is mediated, at least in part, by the opening of the mitochondrial permeability transition pore (PTP) in the first few minutes of re- perfusion and contributes up to $50 \%$ of the final myocardial infarct size. ${ }^{2}$ Crucially, there is currently no effective therapy for preventing myocardial reperfusion injury in patients with STEMI treated by primary PCI.

Inhibiting the opening of the PTP with the use of cyclosporine has been shown to reduce myocardial infarct size in small and large animal models. ${ }^{2}$ Piot et al. ${ }^{3}$ translated this therapeutic approach in patients with STEMI and found a reduction in enzymatic myocardial infarct size with cyclosporine when it was administered before reperfusion. As now reported in the Journal, the same authors have investigated whether this therapeutic approach can improve clinical outcomes in this patient group. ${ }^{4}$

In the multicenter, phase 3 CIRCUS (Does Cyclosporine Improve Clinical Outcome in ST 
Elevation Myocardial Infarction Patients) study, 970 patients with STEMI were administered either an intravenous bolus of cyclosporine (at a dose of $2.5 \mathrm{mg}$ per kilogram of body weight) or placebo control, immediately before reperfusion by means of primary PCI. The primary composite outcome was the incidence of death from any cause, worsening of heart failure after the initial hospitalization, rehospitalization for heart failure, or an increase of $15 \%$ or more in the left ventricular end-diastolic volume (as assessed by means of echocardiography) at 1 year. No significant difference between cyclosporine therapy and placebo control was observed in the primary composite outcome. So why, despite the promising experimental and clinical data, did cyclosporine have no effect on clinical outcomes?

Several factors may be considered. First, although the experimental data supporting the cardioprotective effect of cyclosporine are extensive, not all the studies have been positive. ${ }^{5}$ The clinical evidence in favor of a cardioprotective effect is limited to one study in this patient group. ${ }^{3}$ Another study involving patients with STEMI who were treated with thrombolysis showed no effect with cyclosporine. ${ }^{6}$

Second, the use of an increase in left ventricular end-diastolic volume as one of the primary outcomes has to be queried. This outcome, for which data were missing in $17 \%$ of the patients, is not a clinical one but a surrogate marker of adverse left ventricular remodeling. The high incidence of this outcome in this study (41 to $43 \%$ ) would have made it difficult for a study with a sample of 970 patients to detect a significant difference in the rate of death from any cause, worsening of heart failure after the initial hospitalization, or rehospitalization for heart failure.

Finally, the use of CicloMulsion (NeuroVive Pharmaceutical), a new formulation of cyclosporine that was used in this study, instead of Sandimmune (Novartis), which was used in the original study by Piot et al., ${ }^{3}$ may have contributed to the neutral results. CicloMulsion contains a lipid emulsion carrier vehicle, thereby avoiding the risk of anaphylaxis that has been associated with Sandimmune, which uses an ethanol and polyoxyethylated castor oil (Cremophor EL) carrier vehicle.

The finding that CicloMulsion did not reduce enzymatic myocardial infarct size is problematic and might suggest that this formulation of cyclosporine was ineffective at preventing myocardial reperfusion injury, thereby explaining why it had no effect on clinical outcomes. Although CicloMulsion has been shown to be similar to Sandimmune in terms of bioefficacy, ${ }^{7}$ there appear to be no published experimental or clinical data showing that it can reduce myocardial infarct size. Furthermore, experimental studies in animals have shown that the lipid emulsion vehicle can itself inhibit PTP opening and reduce myocardial infarct size when it is administered at the onset of reperfusion. ${ }^{8}$ As such, the results of the recently completed CYCLE (Cyclosporine A in Reperfused Acute Myocardial Infarction) study (ClinicalTrials.gov number, NCT01650662), which involved 444 patients and used Sandimmune, are eagerly awaited - although in that trial the primary end point was ST-segment resolution rather than clinical outcomes. Curiously, the neutral effects of remote ischemic preconditioning (an endogenous cardioprotective strategy induced by transient limb ischemia and reperfusion) on clinical outcomes after cardiac bypass surgery ${ }^{9}$ have been potentially attributed to the use of propofol, another agent that uses lipid emulsion as the carrier vehicle. ${ }^{10}$

In summary, although the results of the CIRCUS study are disappointing, they do not disprove the existence or clinical significance of myocardial reperfusion injury, because it appears that the formulation of cyclosporine used in the study might not have been effective at preventing myocardial reperfusion injury. If the benefits of PTP inhibition are to be harnessed, more specific inhibitors will need to be discovered. Therefore, the search to find an effective therapy for preventing myocardial reperfusion injury and improving clinical outcomes in patients with reperfused STEMI should continue.

Disclosure forms provided by the authors are available with the full text of this article at NEJM.org.

From the Hatter Cardiovascular Institute, University College London, and the National Institute of Health Research University College London Hospitals Biomedical Research Centre both in London (D.J.H., D.M.Y.); and the Cardiovascular and Metabolic Disorders Program, Duke-National University of Singapore, and the National Heart Research Institute Singapore, National Heart Center Singapore - both in Singapore, Singapore (D.J.H.).

This article was published on August 30, 2015, at NEJM.org.

1. Yellon DM, Hausenloy DJ. Myocardial reperfusion injury. N Engl J Med 2007;357:1121-35. 
2. Ong SB, Samangouei P, Kalkhoran SB, Hausenloy DJ. The mitochondrial permeability transition pore and its role in myocardial ischemia reperfusion injury. J Mol Cell Cardiol 2015;78: 23-34.

3. Piot C, Croisille P, Staat P, et al. Effect of cyclosporine on reperfusion injury in acute myocardial infarction. $\mathrm{N}$ Engl J Med 2008;359:473-81.

4. Cung T-T, Morel O, Cayla G, et al. Cyclosporine before PCI in patients with acute myocardial infarction. N Engl J Med 2015; 373:1021-31.

5. Karlsson LO, Zhou AX, Larsson E, et al. Cyclosporine does not reduce myocardial infarct size in a porcine ischemia-reperfusion model. J Cardiovasc Pharmacol Ther 2010;15:182-9.

6. Ghaffari S, Kazemi B, Toluey M, Sepehrvand N. The effect of prethrombolytic cyclosporine-A injection on clinical outcome of acute anterior ST-elevation myocardial infarction. Cardiovasc Ther 2013;31(4):e34-e39.

7. Ehinger KH, Hansson MJ, Sjövall F, Elmér E. Bioequivalence and tolerability assessment of a novel intravenous ciclosporin lipid emulsion compared to branded ciclosporin in Cremophor EL. Clin Drug Investig 2013;33:25-34.

8. Li J, Iorga A, Sharma S, et al. Intralipid, a clinically safe compound, protects the heart against ischemia-reperfusion injury more efficiently than cyclosporine-A. Anesthesiology 2012; 117:836-46.

9. Hausenloy DJ, Candilio L, Laing C, et al. Effect of remote ischemic preconditioning on clinical outcomes in patients undergoing coronary artery bypass graft surgery (ERICCA): rationale and study design of a multi-centre randomized double-blinded controlled clinical trial. Clin Res Cardiol 2012;101:339-48.

10. Kottenberg E, Thielmann M, Bergmann L, et al. Protection by remote ischemic preconditioning during coronary artery bypass graft surgery with isoflurane but not propofol - a clinical trial. Acta Anaesthesiol Scand 2012;56:30-8.

DOI: 10.1056/NEJMe1509718

Copyright (c) 2015 Massachusetts Medical Society. 